\title{
Crowdsourced Driving Comfort Monitoring
}

\author{
Marcin Badurowicz ${ }^{1 *}$, Jerzy Montusiewicz' ${ }^{1}$ Sławomir Przyłucki ${ }^{1}$ \\ 1 Department of Computer Science, Faculty of Electrical Engineering and Computer Science, Lublin University \\ of Technology, Nadbystrzycka 36B, 20-618 Lublin, Poland \\ * Corresponding author's email: m.badurowicz@pollub.pl
}

\begin{abstract}
In this paper, the authors are showing a calculation of the road quality index called Simple Road Quality Index (SRQI) using the weight provided by the amateur drivers to rate their comfort best possibly on driving on that road. The index is calculated from acceleration data acquired by the smartphone application and is aggregated in a crowdsourcing system for the classification of road quality using the fuzzy membership function. The paper shows that the proposed index correctly shows road quality changes over time and may be used as a way to mark roads to be avoided or needs to be repaired. The numerical experiment was based on the same street in Lublin, Poland, in 2015-2021 and is correctly showing that the quality of analyzed roads deteriorated over time, especially in the winter season.
\end{abstract}

Keywords: road quality, accelerometer, anomaly detection, road artefacts, quality monitoring.

\section{INTRODUCTION}

The problem of finding the most comfortable roads for the drivers, who want to avoid traffic jams to go to their destination easily has one more important aspect - the quality of the road. Because even if users are avoiding traffic jams but are driving over a country road or a road full of potholes or speed bumps, their overall comfort is dropping. The driver should be sure about the road quality he or she wants to drive into and that information may be unavailable without going through in the road.

Current technological advances in smartphone technology allowed to equip of these devices with a set of different environmental sensors: accelerometers, microphones, gyroscopes, video cameras and more. With such a broad range of sensors, there is a possibility to acquire their readings with frequencies defined by firmware or software created for the smartphone platform.

Nowadays, there are over two billion users in the world, and over the course of the last five years, the percentage of smartphone users has risen dramatically [1]. Devices and actual data acquired by the software prepared to acquire data from smartphone sensors have been already used in different community sensing ideas, starting from urban road usage patterns [1], already available also in commercial products, as well as detection of potholes, and even earthquakes [2]. In this paper, the authors are willing to propose a method for describing road quality from the viewpoint of drivers and its classification by fuzzy means and provide a conceptual framework for the crowdsourcing system available to analyze and monitor this information based on data acquired from the smartphone sensors' readings.

The goal of the research was:

- To introduce the Simple Road Quality Index (SRQI) numerical factor for describing the road quality and track their changes over time.

- Use the SRQI to analyze and assess changes in quality in the roads in Lublin, Poland.

\section{Related Works}

The problem of calculating road quality with usage of crowdsourcing and smartphones has been discussed earlier [3, 4], however, most 
researchers are discussing detection of single road artefacts [5], e.g. potholes or speed bumps using different methods [6, 7]. Vibration-based methods [8] are usually compared to video analytics [9]. The crowdsourcing aspects were discussing aggregation and visualization techniques [10], data synergy from multiple sourcing data [11] or proposed different online systems for data aggregation [12]. As for the road quality scoring, usually the IRI (International Roughness Index) is used [13], or its electronic counterpart, eIRI [14, 15]. The IRI is not perfectly understandable for the average road user, so alternative quality indicators have been also proposed [16]. In comparison to the already provided research, this paper concentrates on human-understandable road quality indicators calculation and datapoint aggregation by the usage of polygons and distance windows.

On the other hand, fuzzy logic is being used in this field, due to uncertainty being prevalent in the topic of real-life measurements. The fuzzybased algorithm for road artefacts detection, FTHRESH [17], is one of the components used in the presented research, however other fuzzybased techniques for detection of outliers in time series [18] are also being used in the related research, for example isolation forests [19].

\section{The Crowdsourcing Acquisition System}

In this paper the authors are working with the modified, offline version of the system dubbed "Community Road Artefacts Detection, Identification and Assessment" (CRADIA), already described in [15].

The sensing part of the system, responsible for sending data to the MQTT (Message Query and Telemetry Transport) broker in the online version from which they are being further analyzed in the processing layers, and for the offline recording of smartphone sensors' data was acquiring the set of heterogeneous data:

- Acceleration in the X, Y, and Z-axis of the device, measured in $\mathrm{g}$, unit relative to the Earth's acceleration factor $\left(9.81 \mathrm{~m} / \mathrm{s}^{2}\right)$,

- Acceleration in the global coordinate system: N, E and Z2,

- The current location from the GNSS (Global Navigation Satellite System),

- Current speed (in $\mathrm{m} / \mathrm{s}$ ), magnetic course and time.

All this information is being acquired every 100 milliseconds and saved as a datapoint. However, GNSS system frequency is only about $1 \mathrm{~Hz}$ due to smartphone operating system limitations. The data acquisition procedure was based on the concept already implemented in the previously published research, where smartphones, were mounted in the car in a stable position, which was the only requirement, and in the case of the experiments presented in this paper, it was in the central console. The orientation of the acquisition device in the car was mitigated using the orientation sensor and translation of the data to the global coordinate system consisting of three axes: N, E and Z2, being magnetic north, magnetic east and perpendicular to the Earth's surface, respectively.

In the offline version of the system, recorded datasets were copied from the acquisition devices to PCs, which were running a set of custom Python analysis tools. The first step of the analysis was to calculate the baseline data windows and save them to a database. Data acquired from the smartphone crowdsourcing system will be analyzed in data windows - however in the

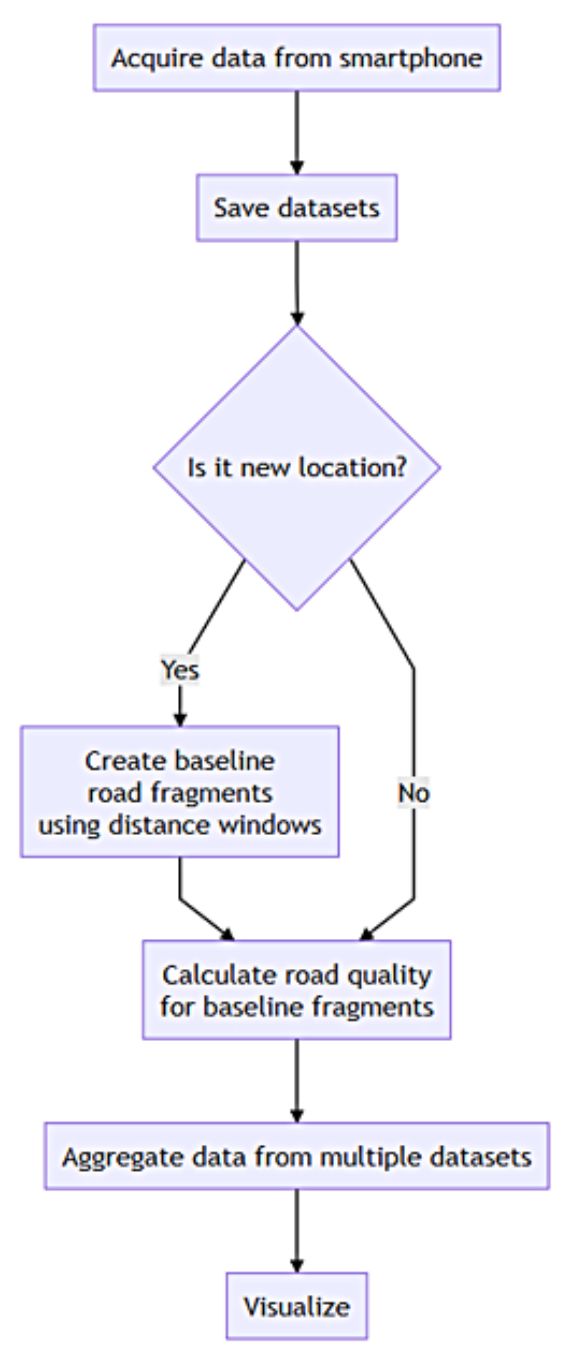

Fig. 1. Flow of data processing in the described system 
presented system windowing will not be based on a number of data packets neither on time, but rather on the physical distance covered by the data points acquired from the smartphone. That is giving two interesting principles: first, all road fragments will be of a similar length. Second, even when the user is staying in a traffic jam and data is being sent to the system over and over from the same geographical position, it won't create additional noise in the system.

Then, the same datasets will be used for driving comfort classification, by numerical experiments described further, finally resulting in a visualization provided using generated HTML documents.

\section{Driving Comfort Classification}

To classify road comfort the scale needs to be created. In this paper, the SRQI will be used, which is being calculated as presented in Figure (1) - as a weighted sum of 4 elements:

$$
S R Q I=R R U I \cdot w_{1}+N D \cdot w_{2} \frac{n}{l} \cdot w_{3}+\left(\sum_{i=1}^{i=n} \chi_{i}\right) \cdot w_{4}
$$

- The first two coefficients, RRUI (Road Relative Unevenness Index) and ND (Normalized Dispersion), are road quality indications previously described in [15] - RRUI is a difference between the analyzed road fragment and the road used as a baseline, while ND is a characteristic of the most problematic elements of the analyzed road fragments,

- The third coefficient, $\mathrm{n} / \mathrm{l}$ is a frequency of road artefacts: $n$ is the number of detected road artefacts after grouping them and 1 is the length of the analyzed road fragment,

- The fourth coefficient is a summed road artefacts scores: $\chi$ is the score of the road artefact as assessed by the recognizing algorithm, giving information about road artefact severity.

All of the proposed coefficients are given weights $w_{1} . w_{4}$ for calculation of the index based on which of these elements of the equation is perceived as the most important, and the actual weights used in the experiment are discussed later.
Still, as pure numerical values are not that interesting for regular drivers, three classes of road fragments were introduced: $\mathrm{A}, \mathrm{B}$ and $\mathrm{C}$, where A is the very good road, B is medium, and $C$ is very bad. The values of SRQI were classified into road quality classes using fuzzy means. The membership function, presented in Figure 2, consisting of two triangular functions for good and bad low quality, starting and ending in the middle of possible values, and gaussian function with mean 0.5 and standard deviation of 0.2 was used for classification. SRQI values were normalized to $0 . .1$ range before classification, based on the minimum and maximum of their values calculated in the experimentation phase, which will be described later.

The maximum membership function value was proposed as a defuzzification measure. The results from the SRQI and membership functions will be used for presentation means: such classification could be changed from the linguistic scale into color scale, for example, green for A-class

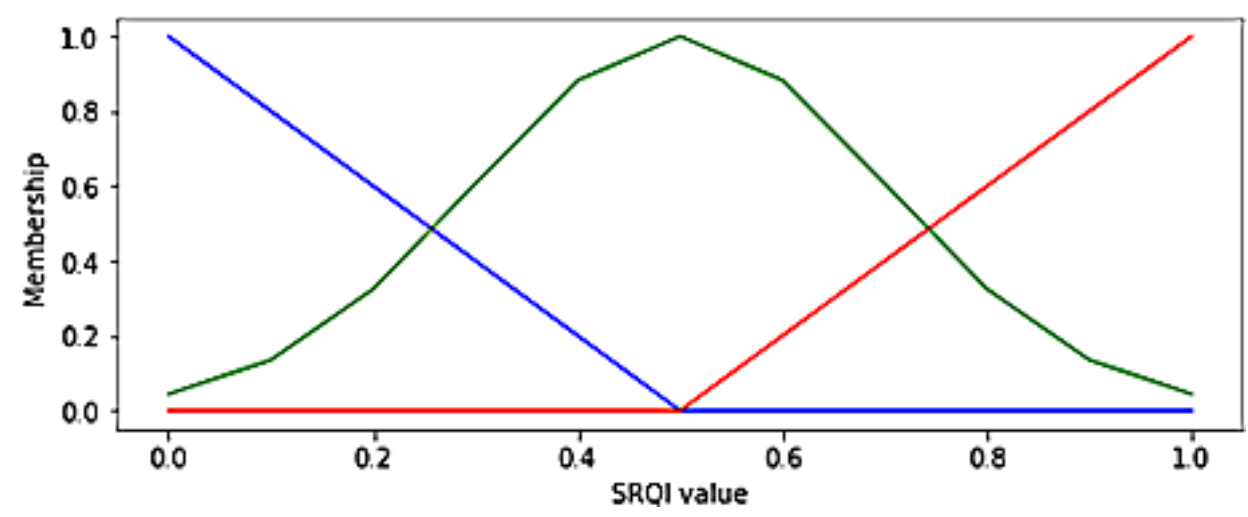

Fig. 2. Fuzzy membership function used for road fragments quality classification 
road, yellow for B-class roads and red for C-class roads, which can be easily presented in the graphical form overlaid on a map.

\section{Deciding on weights}

As mentioned earlier, formula (1) requires weights $w_{l} . w_{4}$ to be known for SRQI road quality classification. To calculate this information possibly best, an on-line questionnaire was presented to a group of 31 amateur drivers.

The questions included in the questionnaire were: the subjective overall state of the roads in the city (in the scale from 1 to 5 , where 1 is the lowest), the satisfaction of the road maintenance by the local territorial government (from 1 to 5), the interest in the on-line system for road quality monitoring (from 1 to 5), the will of participation in the crowdsourcing experiment (from 1 to 5). One closed-answers question was asked - which factor is the most deciding factor when considering the road being low quality with 3 possible answers:

- Overall bad surface,

- Single, but deep potholes,

- The high number of potholes.

The questionnaire was also asking the participants about their age, years of experience in driving, and the amount of driving every year by the number of kilometers driven.

Finally, 34\% of responses said that overall bad surface is the deciding factor, $15 \%$ that single deep pothole, and $51 \%$ that a high number of potholes without describing their depth.

Thus, weights were chosen as:

- $w_{l}$, which is a weight for RRUI being the overall bad road surface, to be 0.34 ,

- $w_{2}$, which is a weight for ND, being the classification how problematic is the single worst road artefact on the road, was chosen to be 0.07 , which is half of $15 \%$,

- $w_{3}$, a frequency of road artefacts, was decided to be 0.51 ,

- $w_{4}$, a severity of road artefacts, was the second half of $15 \%, 0.08$.

Finally, because in all cases: RRUI, ND and summed artefacts score higher value means lower comfort over driving the particular road fragment, the higher SRQI values indicate worse driving conditions.

\section{A method of updating the Simple Road Quality Index using crowdsourcing data}

The SRQI is calculated for the single driving over a particular road fragment, however, road fragments are calculated from the data provided by the user to the crowdsourcing system, thus his or her fragments may not overlap perfectly with data already stored in the database. Such a situation is presented in Figure 3, where four datasets (colored: silver, maroon, teal and olive) are slightly in different positions.

Such a situation is because of two things: first, starting and ending points for separate road fragments calculation were calculating separately over time, so they may start and end in different places. Second, the GNSS system accuracy varies over time due to i.e. atmospheric and geomagnetic variability.

In the previously introduced method [16] based on the overlapping road fragments was possible to create a much fragmentation of the road fragments database, so in this solution the alternative method was used: when the user is the first user to drive over a new road, his or her data is used as a baseline to create road fragments and save their starting and ending positions to a database. Every new user driving over the same

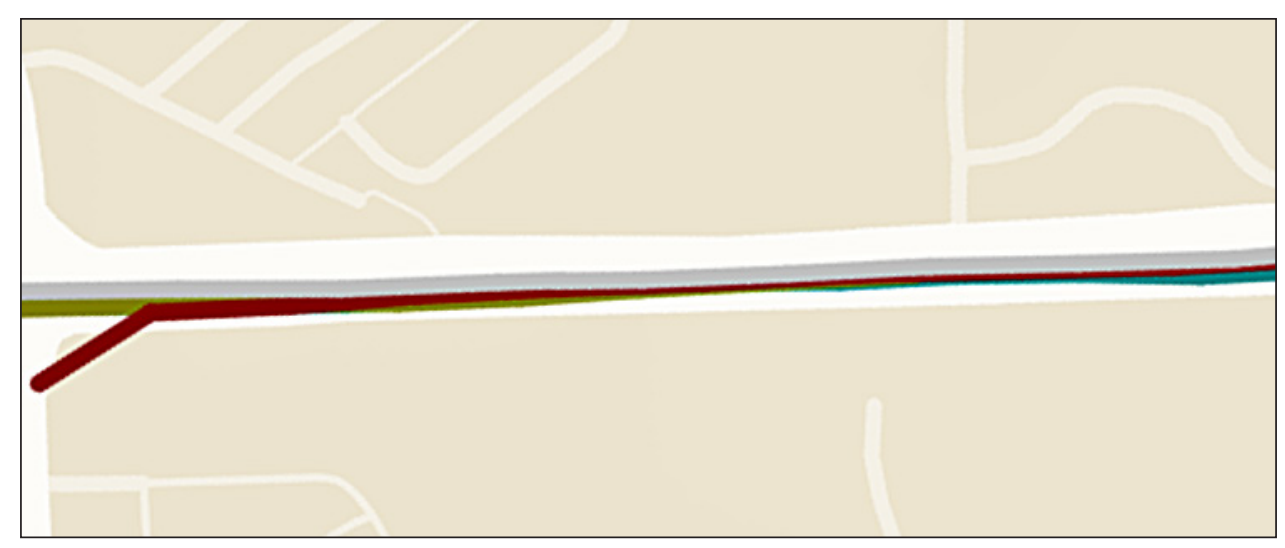

Fig. 3. Partially overlapping readings of 4 users driving over the same road 


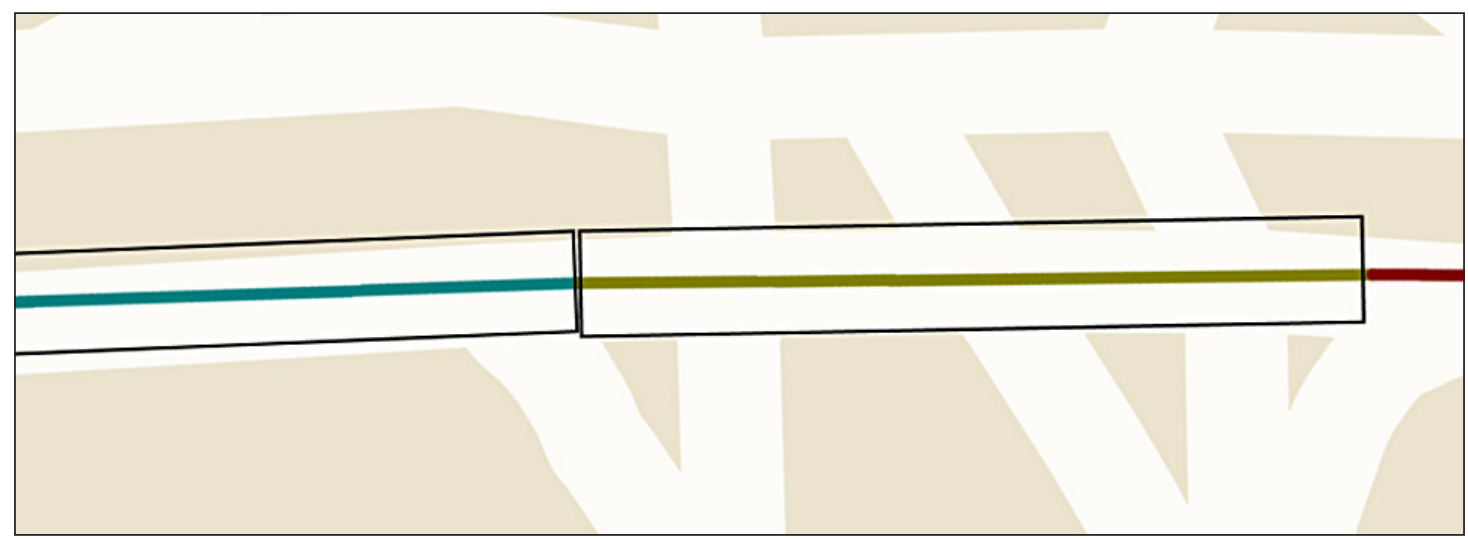

Fig. 4. Constructed membership polygons from the road segments

road will send data where distance windows will be based on the already established road fragments positions. To classify a fresher data point into a baseline road fragment, the virtual "polygon" was used, which centerline was based on a base road fragment's starting and ending point, and its width was decided to be 10 meters, which is an average value of GNSS system accuracy [20]. Such polygons for the two example road fragments are presented in Figure 4. The polygons will be used for classification if the user's provided datapoint is a part of an already existing road fragment and if yes - which one.

The second problem is how to calculate aggregated data from the new drives over the same road fragment - in the previous approach [16], the usage of weighted average was postulated, where "fresh" data was more important than older data, as the road may deteriorate slowly over time before the new user of the proposed computing system will appear, and new data should be always treated as more important than old data.

Thus, the equation to calculate the final SRQI score $\left(\mathrm{SRQI}_{\mathrm{F}}\right.$ ) for the road fragment using data from the new SRQI classification $\left(\mathrm{SRQI}_{\mathrm{N}}\right)$ and older classifications $\left(\mathrm{SRQI}_{1}, \mathrm{SRQI}_{2}, \ldots, \mathrm{SRQI}_{\mathrm{N}-1}\right)$ is:

$$
S R Q I_{F}=\frac{S R Q I_{N}+S R Q I_{N-1} \cdot M^{-T_{N}}+S R Q I_{N-2} \cdot M^{-T_{N-1}}+\cdots}{1+M^{-T_{N}}+M^{-T_{N-1}}+\cdots}
$$

where: $M$ is the constant, defining weight of the older classification, $T_{i}$ is the number of months of difference between a current and i-th measurement on the same road fragment, $N$ is the total number of measurements, $S R Q I_{1}$ is the oldest measurement.

The number of months in $\mathrm{T}$ was chosen because of the fact, that road deterioration is not visible in very short timeframes like weeks or days. Then, the final SRQI value will be reclassified into a threeclasses $\mathrm{A}, \mathrm{B}$, or $\mathrm{C}$ as described earlier, for easier understanding of the road quality for the application user. The formula (2) however raises the question of how much in the past the algorithm should look, as maybe there is such low weight that it will change the final score negligibly however lengthening the calculation process. To cope with an appearing dilemma, experiments were performed, which will be described in the next chapter.

\section{EXPERIMENTAL RESULTS}

The main source for the numerical experiments was the archival data acquired during the extensive experimentation phase in 2015-2021 in Lublin, Poland, previously used in the road artefact detection and identification research. Data was acquired using three cars of different classes during 6 data acquisitions: one in March 2015, two in February 2016 (in the beginning and at the end of the month), in March 2017, in December 2020 and February 2021. The datasets were named "2015", “2016", “2016-2", “2017”, "2020" and " 2021 " by their year of conduct.

The analyzed road was a fragment of Krasnicka Street in Lublin, the south side from the crossing with Głęboka Street until the Honorowych Krwiodawców Roundabout, as one of the most important roads in Lublin, connecting the city with suburbs, with a high traffic every day, including cargo and bus transportation. The final fragments of the road are also equipped with traffic lights.

As for the detection of road artefacts, which is used in the SRQI calculation, the F-THRESH algorithm was used [16]. F-THRESH has an accuracy 


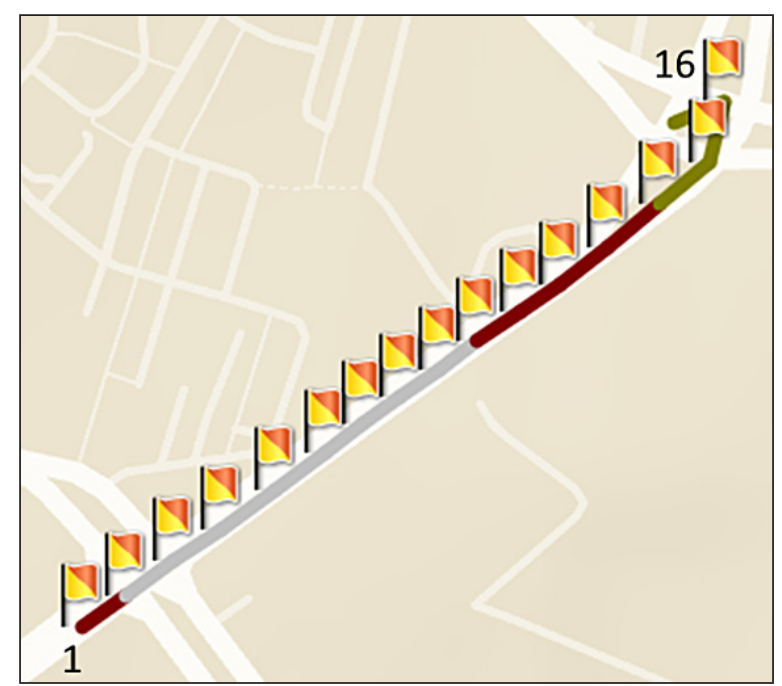

Fig. 5. Starting points of the road fragments as calculated by the authors' system

of over $94 \%$ and a low (less than $2 \%$ ) rate of false positives. The score of the road artefact, aka its severity, was calculated as a difference between the current datapoint's vertical acceleration value and the calculated threshold by the algorithm.

In Figure 5, flags are marking starting points of the calculated road fragments (numbered from 1 to 16 ), each one being 50 meters long, which is a rough estimate of 10 times the average length of the car. The colors are the graphical representation of how much overlapping data are taking part in the scoring of this particular road fragment: olive is 4 , maroon is 5 and gray is 6 . Table 1 presents the scores for all road fragments as calculated by the algorithm, or blank if this road fragment was not available in the dataset.

Using the weights as defined by the questionnaire described earlier, SRQI values for the analyzed road fragments in the experimentation phase were ranging from -1.392 to 7.153 which were used as normalization values, as in the next step SRQI values were normalized to 0..1.

As for the SRQI ${ }_{F}$ calculation, using the SRQI values from all possible measurements, using formula (2), the value of $M$ constant should be determined. If the $M$ is less than 1, older readings will be more important than new ones and for values greater than 1, older readings are less important than fresher ones.

In Tables 2-4, there are compared differences between the last calculated SRQI from 2021 dataset and SRQI calculated using formula (2) with different $M$ values. The T numbers, number of months between dataset acquisition, were: 13 (March 2017 and February 2016), 14 (March 2017 and January 2016), 24 (March 2017 and March 2015), 32 (December 2020 to March 2017) and 2 (February 2021 and December 2020). The difference column shows the difference between the SRQI value calculated using the formula (2) and freshest value (from 2021 dataset).

As presented, the impact of the historical data in all cases very low, even if the case of $\mathrm{M}=1.15$, where the 2-month-old dataset was calculated with a weight of 0.75 , but the next one

Table 1. Normalized SRQI values acquired during the numerical experiments, calculated with weights defined by the questionnaire

\begin{tabular}{|c|c|c|c|c|c|c|}
\hline Number & Dataset 2015 & Dataset 2016 & Dataset 2016-2 & Dataset 2017 & Dataset 2020 & Dataset 2021 \\
\hline 1 & 0.2874170 & 0.1315526 & - & 0.2683063 & 0.2857117 & 0.3754209 \\
\hline 2 & 0.2526824 & 0.0568875 & 0.0494669 & 0.1379621 & 0.4057810 & 0.3365496 \\
\hline 3 & 0.3170474 & 0.1338857 & 0.0689216 & 0.1880392 & 0.2737428 & 0.2391721 \\
\hline 4 & 0.3079526 & 0.0821367 & 0.1308271 & 0.0980328 & 0.2480316 & 0.2725801 \\
\hline 5 & 0.1455556 & 0.0827646 & 0.1196787 & 0.0924068 & 0.2393521 & 0.2591973 \\
\hline 6 & 0.2682149 & 0.0855762 & 0.1163947 & 0.1112350 & 0.2188149 & 0.2005666 \\
\hline 7 & 0.2749327 & 0.0913133 & 0.0858175 & 0.1146354 & 0.2348219 & 0.3379372 \\
\hline 8 & 0.2990742 & 0.0791241 & 0.1398893 & 0.1352919 & 0.3668939 & 0.4180300 \\
\hline 9 & 0.2667193 & 0.1326187 & 0.1247039 & 0.1216839 & 0.2928530 & 0.4102625 \\
\hline 10 & 0.2839866 & - & 0.1183638 & 0.1737087 & 0.2249424 & 0.4076867 \\
\hline 11 & 0.3548288 & - & 0.1399525 & 0.1996479 & 0.3002433 & 0.3870046 \\
\hline 12 & 0.3307673 & - & 0.1541445 & 0.2060008 & 0.4163890 & 0.3762109 \\
\hline 13 & 0.6946293 & - & 0.1290512 & 0.1617379 & 0.5519122 & 0.5259045 \\
\hline 14 & 0.9822782 & - & - & 0.1908586 & 0.1865411 & 0.2342132 \\
\hline 15 & 0.4757146 & - & - & 0.0857266 & 0.0906375 & 0.2279371 \\
\hline 16 & 1.0000000 & - & - & 0.0000000 & & - \\
\hline
\end{tabular}


Table 2. Different $M$ coefficient values and their impact on SQRI factor $(\mathrm{M}=1.15)$

\begin{tabular}{|c|c|c|c|}
\hline Segment & Newest SRQI & SRQI if M=1.15 & Difference \\
\hline 1 & 0.3754209 & 0.3363970 & $-10 \%$ \\
\hline 2 & 0.3365496 & 0.3651792 & $9 \%$ \\
\hline 3 & 0.2391721 & 0.2537000 & $6 \%$ \\
\hline 4 & 0.2725801 & 0.2611814 & $-4 \%$ \\
\hline 5 & 0.2591973 & 0.2498515 & $-4 \%$ \\
\hline 6 & 0.2005666 & 0.2079298 & $4 \%$ \\
\hline 7 & 0.3379372 & 0.2926231 & $-13 \%$ \\
\hline 8 & 0.4180300 & 0.3946850 & $-6 \%$ \\
\hline 9 & 0.4102625 & 0.3584990 & $-13 \%$ \\
\hline 10 & 0.4076867 & 0.3281986 & $-19 \%$ \\
\hline 11 & 0.3870046 & 0.3488697 & $-10 \%$ \\
\hline 12 & 0.3762109 & 0.3925424 & $4 \%$ \\
\hline 13 & 0.5259045 & 0.5351842 & $2 \%$ \\
\hline 14 & 0.2342132 & 0.2135366 & $-9 \%$ \\
\hline 15 & 0.2279371 & 0.1683817 & $-26 \%$ \\
\hline
\end{tabular}

Table 3. Different $M$ coefficient values and their impact on SQRI factor $(\mathrm{M}=1.5)$

\begin{tabular}{|c|c|c|c|}
\hline Segment & Newest SRQI & SRQI if M=1.5 & Difference \\
\hline 1 & 0.3754209 & 0.3478180 & $-7 \%$ \\
\hline 2 & 0.3365496 & 0.3578514 & $6 \%$ \\
\hline 3 & 0.2391721 & 0.2498092 & $4 \%$ \\
\hline 4 & 0.2725801 & 0.2650266 & $-3 \%$ \\
\hline 5 & 0.2591973 & 0.2530910 & $-2 \%$ \\
\hline 6 & 0.2005666 & 0.2061814 & $3 \%$ \\
\hline 7 & 0.3379372 & 0.3062093 & $-9 \%$ \\
\hline 8 & 0.4180300 & 0.4022956 & $-4 \%$ \\
\hline 9 & 0.4102625 & 0.3741363 & $-9 \%$ \\
\hline 10 & 0.4076867 & 0.3514576 & $-14 \%$ \\
\hline 11 & 0.3870046 & 0.3603087 & $-7 \%$ \\
\hline 12 & 0.3762109 & 0.3885733 & $3 \%$ \\
\hline 13 & 0.5259045 & 0.5339066 & $2 \%$ \\
\hline 14 & 0.2342132 & 0.2195448 & $-6 \%$ \\
\hline 15 & 0.2279371 & 0.1856910 & $-19 \%$ \\
\hline
\end{tabular}

Table 4. Different $M$ coefficient values and their impact on SQRI factor $(M=2.0)$

\begin{tabular}{|c|c|c|c|}
\hline Segment & Newest SRQI & SRQI if M=2 & Difference \\
\hline 1 & 0.3754209 & 0.3574791 & $-5 \%$ \\
\hline 2 & 0.3365496 & 0.3503959 & $4 \%$ \\
\hline 3 & 0.2391721 & 0.2460863 & $3 \%$ \\
\hline 4 & 0.2725801 & 0.2676704 & $-2 \%$ \\
\hline 5 & 0.2591973 & 0.2552283 & $-2 \%$ \\
\hline 6 & 0.2005666 & 0.2042162 & $2 \%$ \\
\hline 7 & 0.3379372 & 0.3173142 & $-6 \%$ \\
\hline 8 & 0.4180300 & 0.4078028 & $-2 \%$ \\
\hline 9 & 0.4102625 & 0.3867806 & $-6 \%$ \\
\hline 10 & 0.4076867 & 0.3711379 & $-9 \%$ \\
\hline 11 & 0.3870046 & 0.3696524 & $-4 \%$ \\
\hline 12 & 0.3762109 & 0.3842465 & $2 \%$ \\
\hline 13 & 0.5259045 & 0.5311061 & $1 \%$ \\
\hline 14 & 0.2342132 & 0.2246787 & $-4 \%$ \\
\hline 15 & 0.2279371 & 0.2004772 & $-12 \%$ \\
\hline
\end{tabular}

(32 months older) had the weight of only 0.009 . Thus, the value of M must be correlated with the changes of freshness of data - in the case of frequently used roads in may be lowered, but in case of rarely used - it cannot be very low or the old data will be completely insignificant.

Finally, thanks to the mentioned earlier fuzzy classification into the three classes, the road fragments have been colored with "red", "yellow" and "green", marking their membership in the "poor", "medium" and "good" quality classes, which is presented in Figures 6, 7 and 8.

The question arises how the presented changes in the road classification correspond with real changes on the road noted during the experiment, and if the results are meaningful.

In summer 2015 this particular road fragment was under repair, which means that values from the later datasets should be better than in the first dataset.

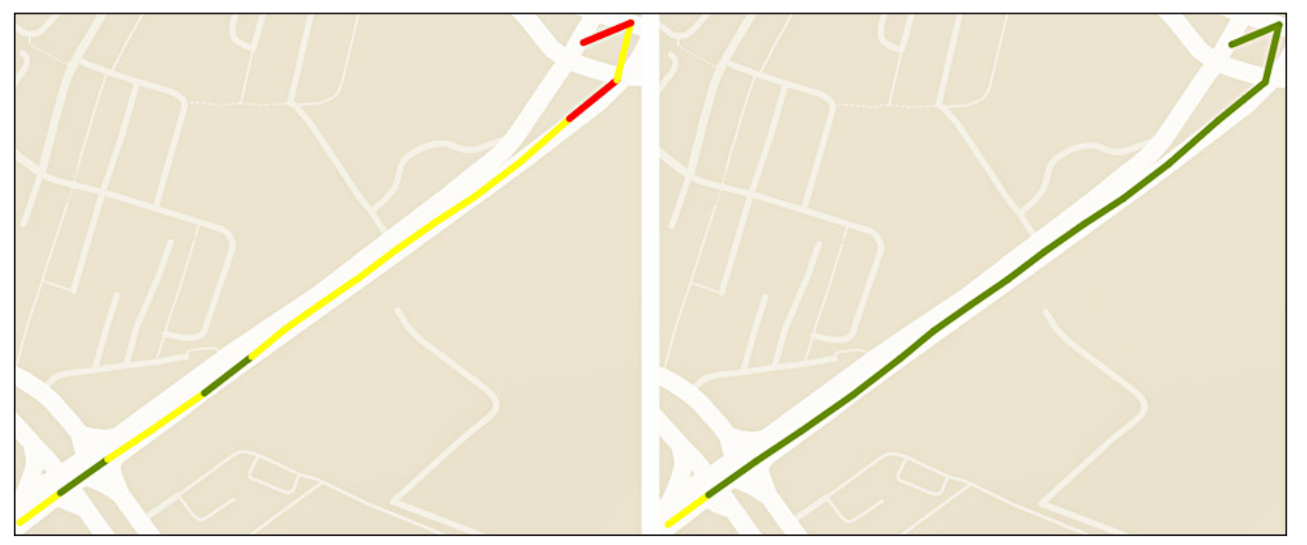

Fig. 6. Visualization of the road quality classification change between year 2015 (left) and 2017 (right) 


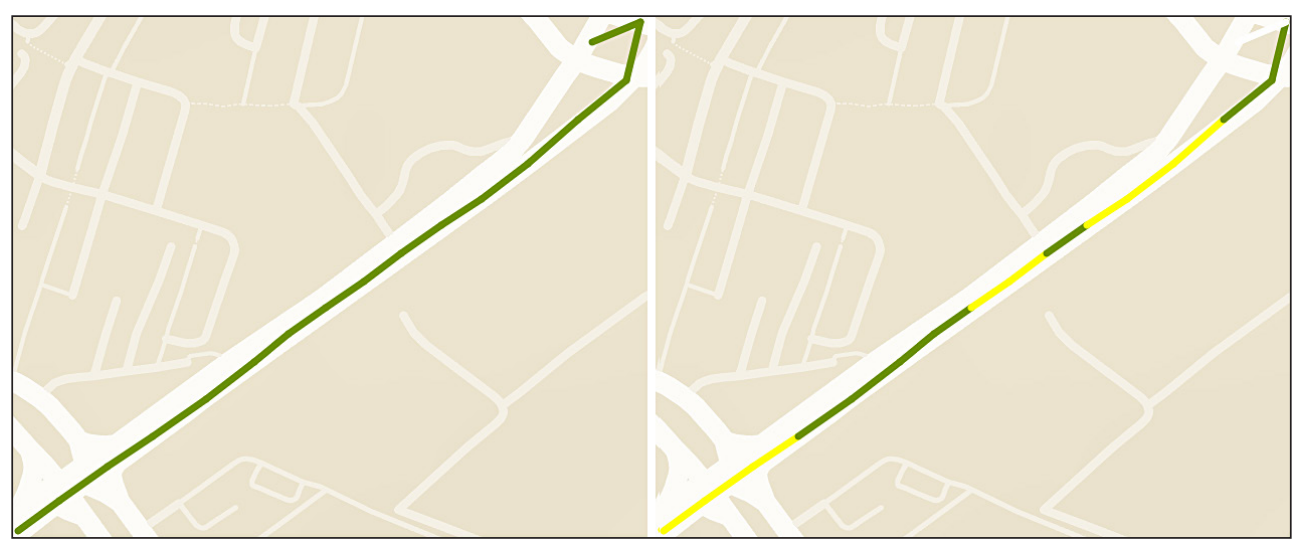

Fig. 7. Visualization of the road quality classification change between year 2017 (left) and 2020 (right)

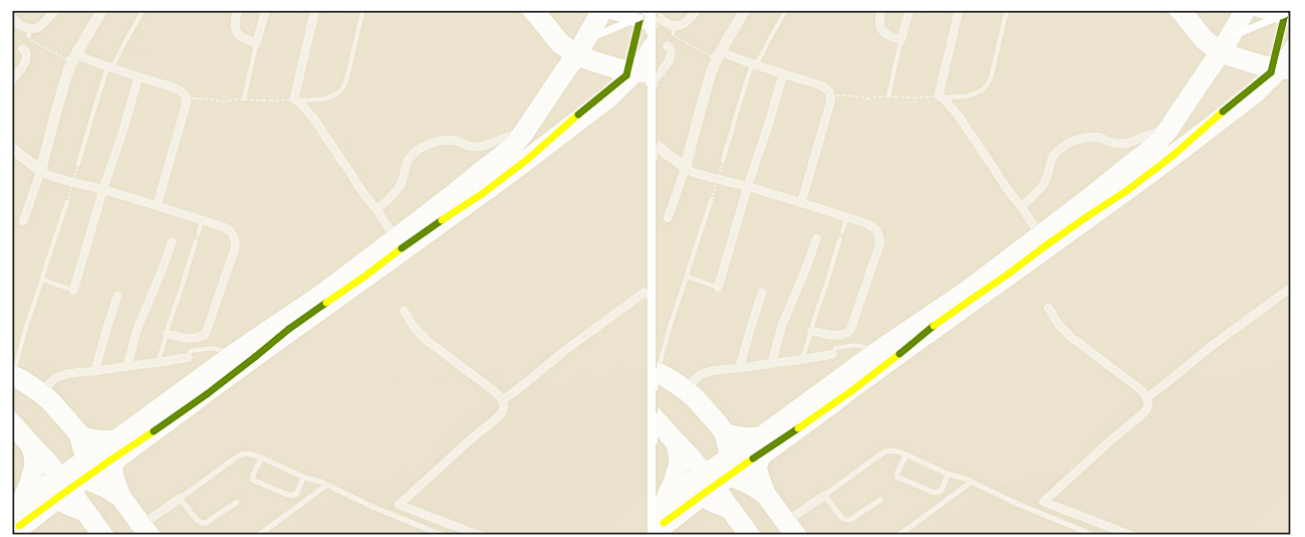

Fig. 8. Visualization of the road quality classification change between year 2020 (left) and 2021 (right)

The normalized values of SRQI from all drives are presented in Table 1 and it is the case, as values in datasets 2016 and 2016-2 are better than in 2015.

As presented in Table 1, in the case of all road fragments, the overall SRQI value has dropped significantly, showing that road quality was indeed improved, while three years later it has risen again showing that the road has been degraded again.

Additionally, one may see that in the cases of fragments $10-13$ as well as $2-3$ the later SRQI scores (from 2017) are worse than scores from the previous year, as there was a slight deterioration of the road quality due to huge amount of traffic on this road, being one of the important communication areas of the city. This shows how sensitive the proposed system is for changes in road quality over time and the visualization of this is presented in Figure 6.

The freshest data of 2020 and 2021 present that in case of many fragments, the road quality deteriorated over the course of only two months. This may indicate the extraordinarily strong road quality changes during the strong winter. The winter of 2020/21 was harsh, with huge temperature differences, especially changes near the freezing point, which is a strong factor in road quality deterioration.

\section{CONCLUSIONS}

Provided research and numerical experiments allows to construct conclusion that the proposed method of heterogeneous data acquisition from the smartphone sensors allows for the acquisition of sufficient data for measuring road quality, which was driven over. The SRQI (Simple Road Quality Index) with weights representing the importance of the four factors and with fuzzy classification method shows road quality in a meaningful way. The numerical experiments have shown that driving over the same road after the repair process significantly changed the road class.

Using the raw SQRI values there may be easily examined that in 2015-2016 the road quality improved, by comparing the SRQI average value of 0.36 in 2015 to 0.1 in 2016, after the repairs, and the road quality deteriorated in the next years, with average SRQI value of 0.14, 0.28 and 0.33 in 2017, 2020 and 2021, respectively, while results from 2021 indicate that the road quality has decreased after a winter with low temperatures and many. 
Results of the questionnaire on the topic of subjective road comfort are that important, as usage of these factors as weights in the SRQI calculation change the calculated quality of the road. Inclusion of user preferences is making numerical results of the real road quality more appropriate to their understanding of the road quality and driving comfort.

Using historical data in the calculation of the road quality index shows that data older than 12 months are very little changing in the road class and may be completely removed in calculations, as the data after 24 months have the weight of only $10^{-5}$, with the $\mathrm{M}$ value equal to 1.15 . Storing and remembering the older SRQI values for the road fragments is however a good idea because of a completely different problem - it is possible to show which roads have been improved in the last years and which are still to be repaired - such data may be interesting for territorial government units.

Finally, prepared quality indices and fuzzy classification methods may be used further in the crowdsourcing road comfort classification system as the authors are planning to build the crowdsourcing system for monitoring road quality, which allows local communities to make a more rational proposal "backed by numbers" for example for participatory budgets for repair the local roads, not monitored globally by the government or territorial governmental units. These solutions will help in more rational money spending on road maintenance and repairing roads where they are really used.

\section{REFERENCES}

1. Matarazzo T., Vazifeh M., Pakzad S., Santi P., Ratti C. Smartphone data streams for bridge health monitoring. Procedia Eng. 2017;199:966-71.

2. Kong Q., Allen R.M., Schreier L., Kwon Y.W.Y.W. MyShake: A smartphone seismic network for earthquake early warning and beyond. Sci Adv. 2016;2(2):e1501055-e1501055.

3. Singh G., Bansal D., Sofat S., Aggarwal N. Smart patrolling: An efficient road surface monitoring using smartphone sensors and crowdsourcing. Pervasive Mob Comput. 2017;40:71-88.

4. Sattar S., Li S., Chapman M. Road surface monitoring using smartphone sensors: A review. Sensors (Switzerland). 2018;18(11).

5. Mednis A., Strazdins G., Zviedris R., Kanonirs G., Selavo L. Real time pothole detection using Android smartphones with accelerometers. 2011 Int Conf Distrib Comput Sens Syst Work DCOSS'11. 2011.
6. Mukherjee A., Majhi S. Characterisation of road bumps using smartphones. Eur Transp Res Rev. 2016;8(2):1-12.

7. Aksamit P., Szmechta M. Distributed, mobile, social system for road surface defects detection. In: ISCIII 2011 - 5th International Symposium on Computational Intelligence and Intelligent Informatics. 2011;37-40.

8. Jo Y., Ryu S. Pothole detection system using a blackbox camera. Sensors (Switzerland). 2015;15(11).

9. Szczodrak M., Grabowski D., Czyżewski A. Employing economical methods for pavement defects estimation. MATEC Web Conf. 2018;231(43):01016.

10. Seraj F., Meratnia N., Havinga P.J.M. An aggregation and visualization technique for crowd-sourced continuous monitoring of transport infrastructures. 2017.

11. Li X, Goldberg DW. Toward a mobile crowdsensing system for road surface assessment. Comput Environ Urban Syst. 2018;(March).

12. Kalim F., Jeong J., Crater I.M.U. A Crowd Sensing Application to Estimate Road Conditions. IEEE Access. 2016;PP(99):8317-8326.

13. Johannesson P., Rychlik I. Modelling of road profiles using roughness indicators. Int J Veh Des. 2014;66(4):317.

14. Rajamohan D., Gannu B., Maargha R.K. A Prototype System for Road Condition and Surface Type Estimation by Fusing Multi-Sensor Data. ISPRS Int J Geo-Information. 2015;4(3).

15. Aydın M.M., Yıldırım M.S., Forslof L. The Use of Smart Phones To Estimate Road Roughness: a Case Study in Turkey. E-Journal New World Sci Acad. 2018;13(3):247-257.

16. Badurowicz M., Cieplak T. Real-Time Road Quality Assessment Using Smartphones and Cloud Lambda Architecture. Kulisz M., Szala M., Badurowicz M., Cel W., Chmielewska M., Czyż Z., et al., editors. MATEC Web Conf. 2019 Jan 14;252:03011.

17. Badurowicz M., Montusiewicz J., Karczmarek P. Detection of Road Artefacts Using Fuzzy Adaptive Thresholding. In: 2020 IEEE International Conference on Fuzzy Systems (FUZZ-IEEE). 2020.

18. Kiersztyn A., Karczmarek P., Lopucki R., Pedrycz W., Al E., Kitowski I., et al. Data Imputation in Related Time Series Using Fuzzy Set-Based Techniques. In: 2020 IEEE International Conference on Fuzzy Systems (FUZZ-IEEE). 2020.

19. Karczmarek P., Kiersztyn A., Pedrycz W. Fuzzy SetBased Isolation Forest. In: 2020 IEEE International Conference on Fuzzy Systems (FUZZ-IEEE). 2020.

20. Wajszczak E., Galas D. Egnos - Use Of Gps System For Approach Procedures. Adv Sci Technol Res J. 2013;7(17):62-5. 